\title{
Article \\ A Numerical Investigation of a Plasmonic Sensor Based on a Metal-Insulator-Metal Waveguide for Simultaneous Detection of Biological Analytes and Ambient Temperature
}

\author{
Nikolay L. Kazanskiy ${ }^{1,2}$, Svetlana N. Khonina ${ }^{1,2} \mathbb{D}^{\text {, Muhammad A. Butt }}{ }^{1,3, * \mathbb{C}}$, Andrzej Kaźmierczak ${ }^{3}$ \\ and Ryszard Piramidowicz ${ }^{3}$ (D) \\ 1 Samara National Research University, 443086 Samara, Russia; kazanskiy@ipsiras.ru (N.L.K.); \\ khonina@ipsiras.ru (S.N.K.) \\ 2 Institute of RAS-Branch of the FSRC “Crystallography and Photonics" RAS, 443001 Samara, Russia \\ 3 Institute of Microelectronics and Optoelectronics, Warsaw University of Technology, Koszykowa 75, \\ 00662 Warszawa, Poland; andrzej.kazmierczak@pw.edu.pl (A.K.); ryszard.piramidowicz@pw.edu.pl (R.P.) \\ * Correspondence: butt.m@ssau.ru
}

\section{check for} updates

Citation: Kazanskiy, N.L.; Khonina, S.N.; Butt, M.A.; Kaźmierczak, A.; Piramidowicz, R. A Numerical Investigation of a Plasmonic Sensor Based on a Metal-Insulator-Metal Waveguide for Simultaneous Detection of Biological Analytes and Ambient Temperature. Nanomaterials 2021, 11, 2551. https://doi.org/ 10.3390/nano11102551

Academic Editor: Alexander Kromka

Received: 1 September 2021

Accepted: 27 September 2021

Published: 29 September 2021

Publisher's Note: MDPI stays neutral with regard to jurisdictional claims in published maps and institutional affiliations.

Copyright: (c) 2021 by the authors. Licensee MDPI, Basel, Switzerland. This article is an open access article distributed under the terms and conditions of the Creative Commons Attribution (CC BY) license (https:/ / creativecommons.org/licenses/by/ $4.0 /)$.

\begin{abstract}
A multipurpose plasmonic sensor design based on a metal-insulator-metal (MIM) waveguide is numerically investigated in this paper. The proposed design can be instantaneously employed for biosensing and temperature sensing applications. The sensor consists of two simple resonant cavities having a square and circular shape, with the side coupled to an MIM bus waveguide. For biosensing operation, the analytes can be injected into the square cavity while a thermo-optic polymer is deposited in the circular cavity, which provides a shift in resonance wavelength according to the variation in ambient temperature. Both sensing processes work independently. Each cavity provides a resonance dip at a distinct position in the transmission spectrum of the sensor, which does not obscure the analysis process. Such a simple configuration embedded in the single-chip can potentially provide a sensitivity of $700 \mathrm{~nm} / \mathrm{RIU}$ and $-0.35 \mathrm{~nm} /{ }^{\circ} \mathrm{C}$ for biosensing and temperature sensing, respectively. Furthermore, the figure of merit (FOM) for the biosensing module and temperature sensing module is around 21.9 and 0.008 , respectively. FOM is the ratio between the sensitivity of the device and width of the resonance dip. We suppose that the suggested sensor design can be valuable in twofold ways: (i) in the scenarios where the testing of the biological analytes should be conducted in a controlled temperature environment and (ii) for reducing the influence on ambient temperature fluctuations on refractometric measurements in real-time mode.
\end{abstract}

Keywords: plasmonic sensor; metal-insulator-metal waveguide; temperature sensor; refractive index sensor; polydimethylsiloxane

\section{Introduction}

Over the past few years, plasmonic-based devices have gathered significant interest thanks to their unique properties, enabling significant enhancement of the sensitivity of photonic sensors [1,2]. Continuous advancements in nanofabrication alter expectations of the role of metals in the development of new optical devices, based on plasmonic effects, like surface plasmon polaritons (SPPs). These are attractive for use in nanophotonics, biosensing, electronics, imaging, and many other disciplines [3]. SPPs are firmly connected to metal-dielectric interfaces, reaching a depth of around $10 \mathrm{~nm}$ in metal (skin depth) and often more than $100 \mathrm{~nm}$ in dielectric material. The metal-insulator-metal (MIM) waveguide system is one of the most widely utilized plasmonic-based nanostructures for the implementation in integrated photonic circuits $[4,5]$. Such waveguides are plasmonic structures of a simple design, with two metal claddings surrounding an insulator, able to confine SPP mode at the subwavelength level. So far, numerous optical devices developed with this waveguide configuration have been widely investigated, for applica- 
tions such as biosensensing [6-8], temperature sensing [9,10], optical signal switching [11], demultiplexing [12], splitting [5], and filtering [13].

Plasmonic sensors are extremely attractive and in great demand owing to their small footprint and high sensing capabilities as compared with sensors based on other platforms such as silicon photonics or optical fiber $[1,14]$. Because of their ability to overcome the diffraction limit of light, SPP waveguide structures, particularly MIM waveguides, have attracted much attention, with expectations of realizing highly integrated optical circuits owing to their small footprint, ease of integration, and good balance between light localization and transmission loss [15]. A biosensing semiconductor nanowire refractive index sensor with a sensitivity of $235 \mathrm{~nm} / \mathrm{RIU}$ has been demonstrated [16]. In ref. [17], an optical fiber refractive index sensor based on long-period fiber grating has been suggested. The maximum experimental sensitivity for quasi-TM ring resonators was $135 \mathrm{~nm} / \mathrm{RIU}$, according to $\mathrm{Xu}$ et al. [18]. The bulk sensitivity of $270 \mathrm{~nm} / \mathrm{RIU}$ was displayed by adjusting the waveguide thickness [19].

Sensing is a hotspot among several interesting topics, and in the previous years, several plasmonic sensor designs based on MIM waveguides have been numerically investigated and proposed for refractive index and temperature sensing [20-22]. Although the sensitivity of these devices is relatively high, the proposed designs support only one application (either temperature or refractive index sensing) at a time. What is more, the manufacturing of these designs is challenging without leaving an error of a few nanometers owing to their additional complex geometric features. This may lead to a deterioration in the sensing performance. Our aim is not to criticize any previously developed sensor models; however, we would like to demonstrate that promising results can be achieved using a simple, multipurpose, and easy to implement sensor model. A few previously proposed complicated sensor designs used for refractive index and temperature sensing applications are shown in Figure 1a-g [9,23-28].

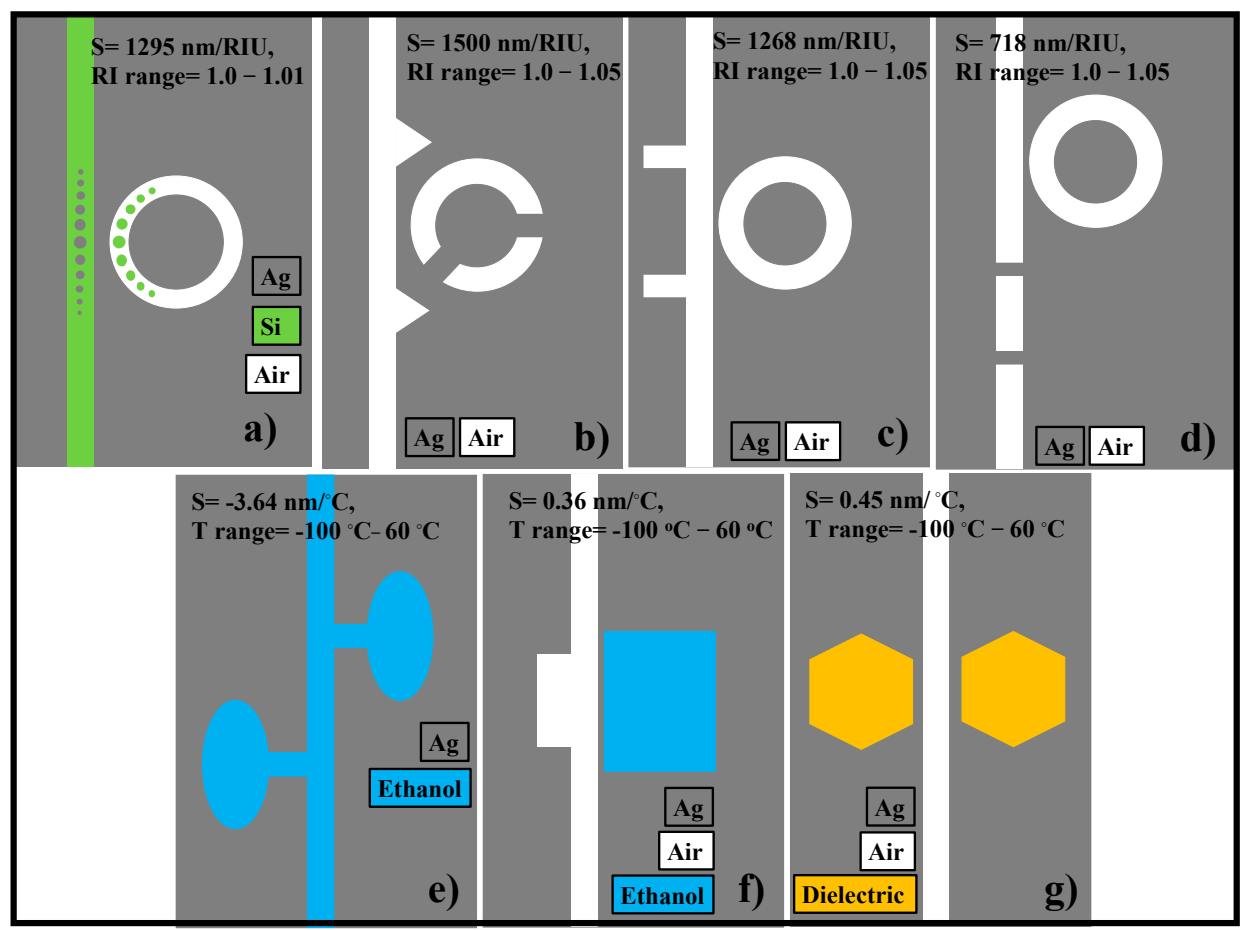

Figure 1. Schematic representation (top view) of single-purpose plasmonic sensor designs found in the literature. Refractive index sensor, (a) ring resonator coupled to an MIM waveguide containing tapered defects [24], (b) two triangle stubs coupled with a split-ring nanocavity [25], (c) two stubs and one ring resonator [26], and (d) two baffles and a coupled ring cavity [27]. Temperature sensors, (e) ethanol-sealed asymmetric ellipse resonators [9], (f) ethanol filled resonator cavity [23], and (g) dual laterally side-coupled hexagonal cavities [28]. 
In ref. [24], the plasmonic sensor that combines a ring resonator containing circular tapered defects coupled to a MIM waveguide with tapered defects for refractive index sensing is proposed. The sensitivity of the device is around $1295 \mathrm{~nm} / \mathrm{RIU}$; however, the device design is so complicated that even a fabrication error of a few nanometers can deteriorate the device performance [24]. In ref. [25], another complicated refractive index sensor comprised of an MIM waveguide with symmetric two triangle stubs coupled to a circular split-ring resonator cavity is proposed. The sensitivity of the device is $1500 \mathrm{~nm} / \mathrm{RIU}$. However, for this sensor configuration, several parameters must be carefully optimized to obtain maximum sensing performance. A similar situation occurs with the designs proposed in ref. [26,27]. The numerical results presented in these studies seem to be attractive; however, the real challenge appears at the stage of fabrication of these devices where several variables must be optimized at a nanometer scale.

It has been recently demonstrated that the MIM plasmonic waveguide structures combined with thermal sensing media such as ethanol or PDMS can be efficiently utilized for temperature sensing applications [10,29-31]. Zhu et al. [9] proposed a sensor design that can only be used for temperature sensing and offers a very high sensitivity of $-3.64 \mathrm{~nm} /{ }^{\circ} \mathrm{C}$. Furthermore, Zhu et al. numerically investigated a compact Fano resonance temperature sensor by utilizing polydimethylsiloxane (PDMS)-sealed semi-square ring resonator. PDMS is a mineral-organic polymer of the siloxane family. It is a polymeric organosilicon chemical that is commonly referred to as silicone. The silicone-based organic polymer PDMS is the most widely used. It is well-known for its distinct features-transparency over a wide wavelength range, the refractive index lower than in fused silica, good elasto-optic and thermo-optic coefficients, biocompatibility, and negligible absorption loss, to enumerate a few [32]. It also has a good mechanical property owing to low Young's modulus; it is soft and deformable with no shrinkage. What is also important from the point of view of mass production is that the manufacturing process is cost-efficient and straightforward. Owing to the high thermo-optic coefficient of PDMS, the devices based on it are highly sensitive to temperature fluctuations. The sensor might be useful for temperature monitoring applications that need a high level of sensitivity. The sensitivity is around $-4 \mathrm{~nm} /{ }^{\circ} \mathrm{C}$; however, the cavity shape is so complex that at least 5-6 parameters should be optimized to obtain the best sensing performance [10]. This restricts the flexibility in the fabrication process. Kong et al. proposed a temperature sensor utilizing ethanol in a resonant cavity providing a sensitivity of $0.36 \mathrm{~nm} /{ }^{\circ} \mathrm{C}$ [23].

In diagnostic centers, the testing of the biological samples requires a controlled temperature environment for accurate results. Therefore, it is required to have a lab-on-chip solution that can examine the analytes and temperature at the same time. In this work, an attractive and straightforward sensor model is proposed that can be simultaneously employed as a biosensor and temperature sensor. The device contains two cavities, each of which is designated for a particular purpose. The resonance dips from both cavities are well separated, which makes evaluating their sensing performance extremely easy. The study is conducted via the 2D-finite element method (2D-FEM) by utilizing COMSOL Multiphysics software. The sensor design has no peculiar geometric features like stub [33] or nanodots [34], which can produce multiple resonance peaks/dips as well as add complications to the manufacturing process. Therefore, we believe that the proposed sensor design will be quite useful in realizing a multipurpose compact lab-on-chip sensor.

\section{Single-Purpose Device Model and Simultaneous Parameters}

In this section, the optimization of the geometric parameters of the square and circular cavity is carried out utilizing the 2D-FEM to determine the transmission spectrum of the device. The 2D model is preferred over the $3 \mathrm{D}$ one because it reduces the processing time and provides accurate results, as demonstrated in other works [24,34-36]. The geometry of the proposed sensor design is straightforward and does not involve any stub $[19,27,37]$, nanodots [24,38], or other complex structures [36] that can complicate the fabrication 
process. The cavities are simple airholes that can be precisely etched with current fabrication technology [39].

The real part of the effective refractive index $\left(\operatorname{Re}\left(\mathrm{n}_{\mathrm{eff}}\right)\right)$ is plotted for the MIM waveguide heights $(H)$ in the range of $50 \mathrm{~nm}$ to $500 \mathrm{~nm}$ at an operational wavelength of $1000 \mathrm{~nm}$. The MIM waveguide is composed of gold $(\mathrm{Au})$ and deposited on a quartz substrate. The waveguide widths of $50 \mathrm{~nm}, 60 \mathrm{~nm}, 75 \mathrm{~nm}$, and $100 \mathrm{~nm}$ are also considered, as shown in Figure 2. In the case of $H=50 \mathrm{~nm}$ and $W=50 \mathrm{~nm}, \operatorname{Re}\left(\mathrm{n}_{\mathrm{eff}}\right)$ is at 2.11 , which decreases and stabilizes to 1.98 as $H$ approaches $300 \mathrm{~nm}$ and onward. The same trend follows for the MIM waveguide with $W=60 \mathrm{~nm}, 75 \mathrm{~nm}$, and $100 \mathrm{~nm}$. The inset of Figure 2 shows the H-field distribution at $H=50 \mathrm{~nm}, 300 \mathrm{~nm}$, and $500 \mathrm{~nm}$. Therefore, from this analysis, we can say that $H=300 \mathrm{~nm}$ or more can be used for the practical realization of the MIM waveguides.

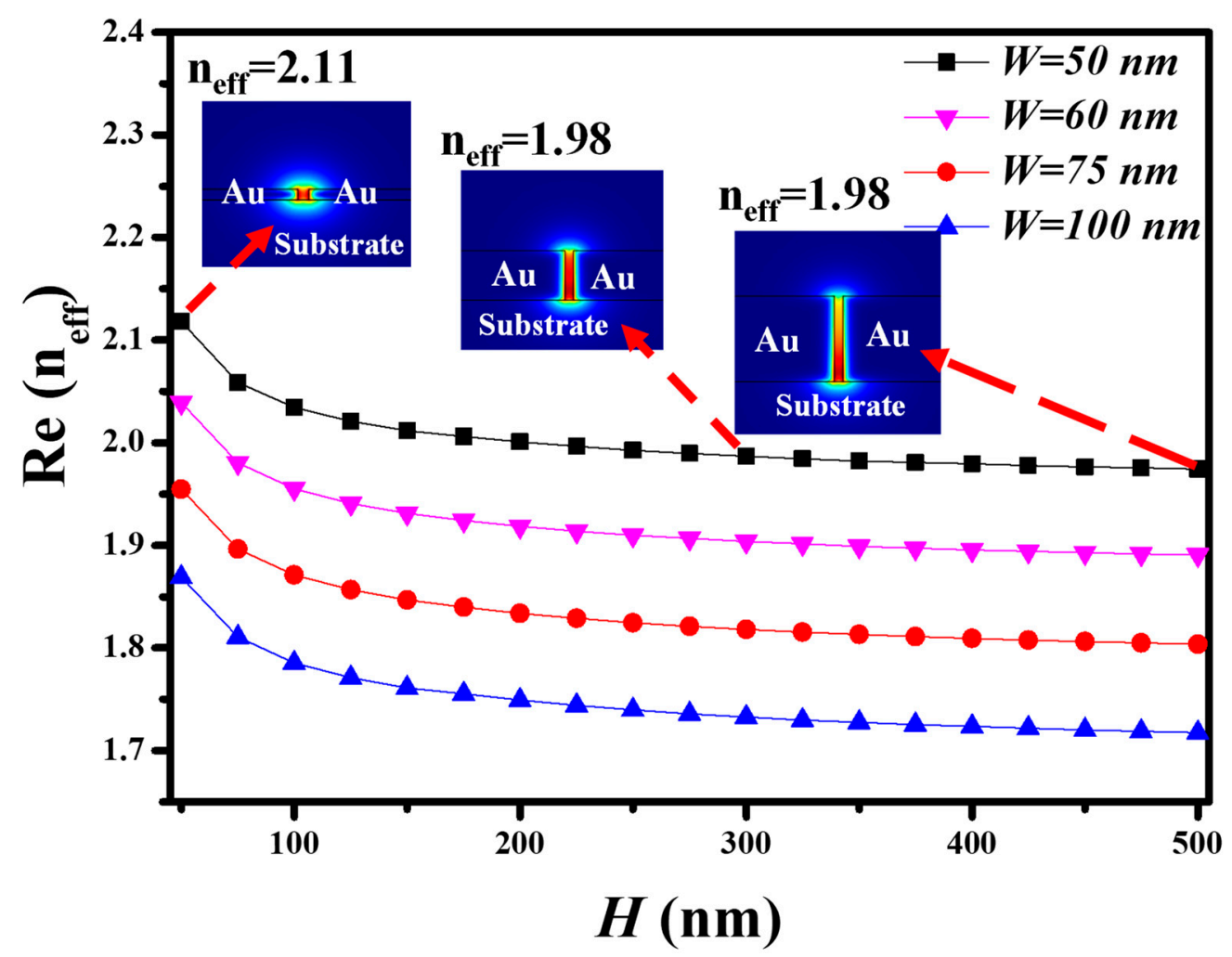

Figure 2. The real part of the effective refractive index versus the height $(H)$ and slot width $(W)$ of the MIM waveguide. Inset shows the cross-section E-field distribution in the MIM waveguide.

We aim to employ a square cavity and circular cavity on the same chip for biosensing and temperature sensing simultaneously. The device structure is composed of an Au layer deposited on a quartz substrate and the MIM waveguide configuration and cavities can be precisely created with the help of electron-beam lithography. However, at first, we would like to individually explore their spectral characteristics. The dielectric material of refractive index $(n)=1.33$, which can be considered as water is filled in the square cavity and is side coupled to an MIM bus waveguide. The width of the MIM waveguide is denoted as $W$, which is fixed at $50 \mathrm{~nm}$ throughout this paper. The side length of the square cavity is represented as $L$ and the gap between the bus MIM waveguide and the cavity is designated as $g$. Au is used as a plasmonic material because of its resistance to oxidation and biocompatibility. The relative dielectric constant of the Au is defined by the Drude-Lorentz dispersion model:

$$
\varepsilon=\varepsilon_{\infty}-\frac{\omega_{p}^{2}}{\omega^{2}+j \omega \gamma}
$$


where $\varepsilon_{\infty}=9.0685, \omega_{p}=135.44 \times 10^{14} \mathrm{rad} / \mathrm{s}$, and $\gamma=1.15 \times 10^{14} \mathrm{rad} / \mathrm{s}$ [40]. The circular cavity is filled with PDMS material, which has a high thermo-optic coefficient. The cavity is separated from the bus waveguide with a small gap, which is represented as $g_{1}$. The radius of the circular cavity is denoted as $R$. The fundamental mode that an MIM waveguide supports is an even mode with a transverse-magnetic (TM) polarization. As a result, an SPP mode is excited, which travels along the metal-dielectric boundary and couples to the designated cavity when the resonance condition is met $[5,26,36,41,42]$. The dispersion relation of the fundamental mode is defined as follows:

$$
\begin{gathered}
\frac{\varepsilon_{i} p}{\varepsilon_{m} k}=\frac{1-e^{k W}}{1+e^{k W}}, \\
k=k_{o} \sqrt{\left(\frac{\beta_{s p p}}{k_{o}}\right)^{2}-\varepsilon_{i}}, p=k_{o} \sqrt{\left(\frac{\beta_{s p p}}{k_{o}}\right)^{2}-\varepsilon_{m},} \\
\beta_{s p p}=n_{e f f} k_{o}=n_{e f f} \frac{2 \pi}{\lambda},
\end{gathered}
$$

where $W, \lambda, \varepsilon_{i}$, and $\varepsilon_{m}$ denote the width of the bus waveguide, incident wavelength in vacuum, the relative dielectric, and metal permittivity, respectively. $n_{\text {eff, }} \beta_{\text {spp }}$, and $k_{o}=2 \pi / \lambda$ are the effective refractive index, the propagation constant of SPPs, and the wave number, respectively. The schematic representation of the plasmonic sensors with a square cavity and a circular cavity is shown in Figure $3 a, b$, respectively.

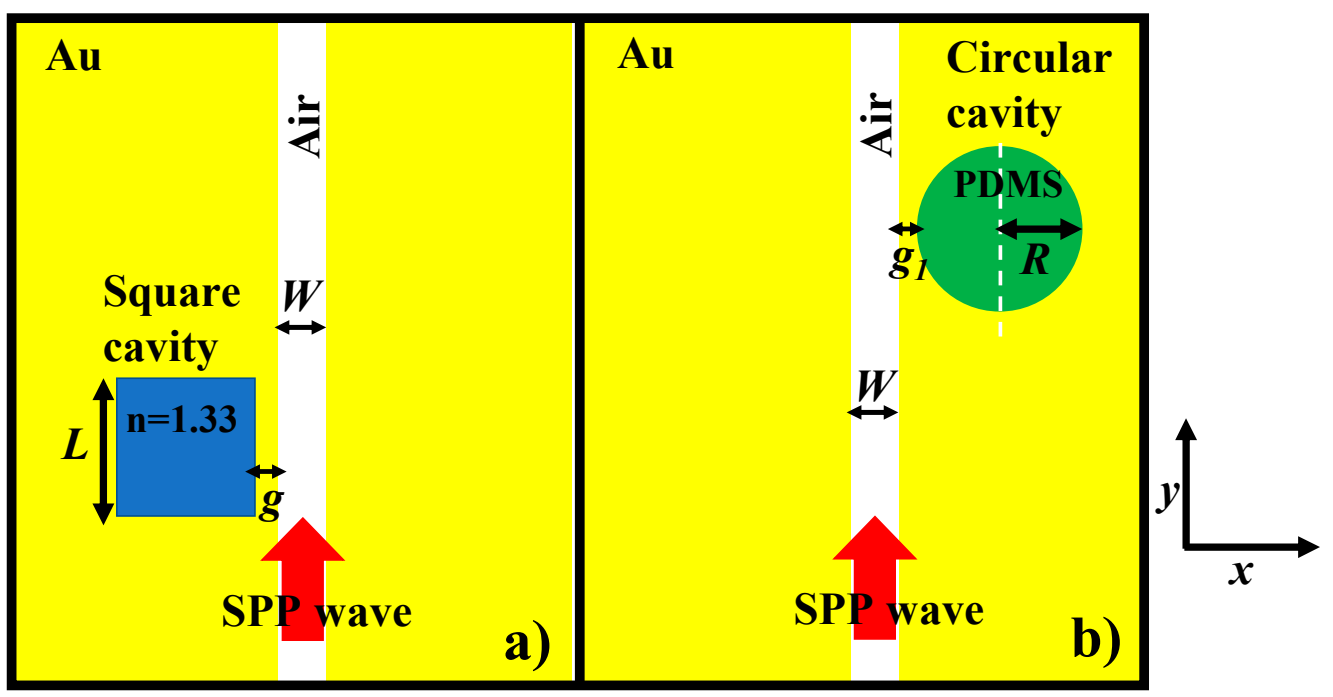

Figure 3. Schematic representation (top view) of the plasmonic cavity coupled to a bus waveguide: (a) square cavity filled with dielectric medium, $n=1.33$, and (b) circular cavity filled with thermo-optic material, PDMS.

The single-purpose device arrangement is so simple that only two variables should be optimized. For the square cavity, the influence of $L$ on $\lambda_{\text {res_s }}$ is determined at $g=20 \mathrm{~nm}$, as shown in Figure $4 \mathrm{a}$. It can be seen that $\lambda_{\text {res_s }}$ performs a redshift as $L$ increases from $280 \mathrm{~nm}$ to $340 \mathrm{~nm}$, following a linear trend. The inset of Figure 4 a shows the $\mathrm{H}$-field distribution at the corresponding resonance wavelength. It is also important to determine the optimum coupling distance between the bus waveguide and the cavity to obtain the maximum coupling power (CP) and highest extinction ratio (ER). The transmission spectrum is plotted for the square cavity of $L=300 \mathrm{~nm}$ at $g=10 \mathrm{~nm}$ to $30 \mathrm{~nm}$, as shown in Figure $4 \mathrm{~b}$. The maximum CP of $>99 \%$ and highest ER of $-18.6 \mathrm{~dB}$ are obtained for $g=20 \mathrm{~nm}$. 



Figure 4. Spectral characteristics of square and circular cavity: (a) $\lambda_{\text {res_s }}$ versus $L$, (b) effect of $g$ on transmission, (c) $\lambda_{\text {res_C }}$ versus $R$, and (d) effect of $g_{1}$ on transmission.

For the circular cavity, $R$ and $g_{1}$ should be optimized for better CP and ER. The PDMS filled cavity is maintained at $g_{1}=20 \mathrm{~nm}$ and ambient temperature of $10^{\circ} \mathrm{C}$. The influence of $R$ on $\lambda_{\text {res_C }}$ is plotted in Figure 4c. Like a square cavity, $\lambda_{\text {res_C }}$ performs a redshift as $R$ increases from $280 \mathrm{~nm}$ to $340 \mathrm{~nm}$ in a linear fashion. Similarly, the coupling distance $g_{1}$ is optimized by maintaining $R=300 \mathrm{~nm}$, as shown in Figure $4 \mathrm{~d}$. The maximum CP and ER of $>80 \%$ of $-7.6 \mathrm{~dB}$ are obtained when $g_{1}=10 \mathrm{~nm}$, respectively. The inset of Figure $4 \mathrm{~d}$ shows the E-field distribution at on-resonance and off-resonance states when $g_{1}=10 \mathrm{~nm}$.

\section{Dual-Purpose Sensor Design}

The schematic representation of the proposed sensor design for the simultaneous detection of biological analytes and temperature is shown in Figure 5a. The square cavity is filled with bio-analytes, whereas the circular cavity is filled with PDMS material. The variation in the refractive index of the PDMS layer concerning the temperature can be expressed as follows [43]:

$$
\mathrm{n}_{\mathrm{PDMS}}(\mathrm{T})=1.4176-4.5 \times 10^{-4} \cdot \mathrm{T},
$$

where $\mathrm{T}$ is the ambient temperature. Both the cavities are at a distinct location and filled with dielectric material of different refractive indices which result in two separated resonance dips at unique positions in the spectrum (see Figure $5 b$ ). The first resonance dip is denoted as $\lambda_{\text {res_S}}$, which corresponds to the square cavity. When the unknown analytes of refractive index higher than 1.33 are introduced into the cavity, $\lambda_{\text {res_s }}$ performs a redshift owing to the variation in the effective refractive index, whereas $\lambda_{\text {res_C }}$ is the second dip in the spectrum, which is connected to the circular cavity. The refractive index of the PDMS versus ambient temperature plot is shown in Figure $5 c$, which shows a linear relation between refractive index and temperature. The reduction in the refractive index of the PDMS layer due to the increase of the ambient temperature results in a blueshift of $\lambda_{\text {res_C }}$. The operation of the sensor is based on the detection of a shift in resonance dips. It is worth noting that the change in $\lambda_{\text {res_s }}$ does not affect $\lambda_{\text {res_C }}$, and vice versa, because both 
the dips are well separated. This indicates that both the cavities work independently and any change in one cavity (in terms of refractive index) does not bring any change in the other. This feature encourages the realization of such devices that can act as biosensors and temperature sensors at the same time.
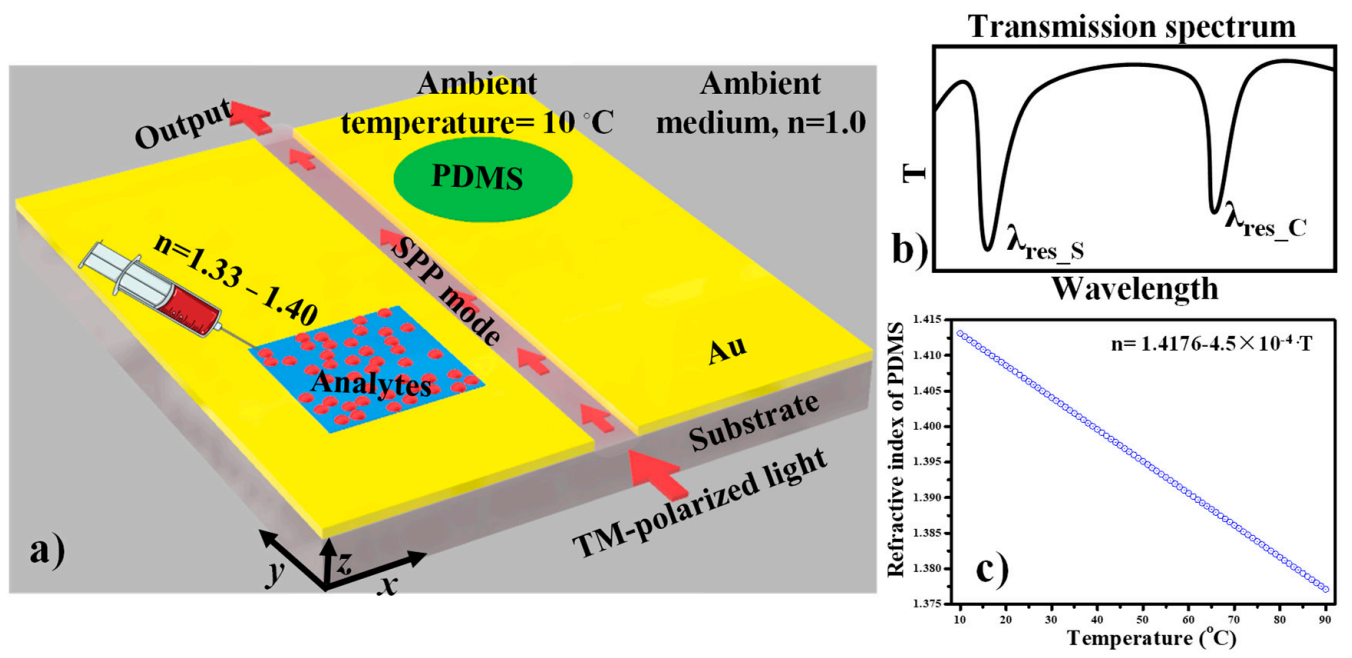

Figure 5. (a) Schematic representation (3D view) of a plasmonic sensor for simultaneous sensing of biological analytes and temperature, (b) The intended transmission spectrum of the proposed device, (c) Variation in the refractive index of the PDMS material versus the ambient temperature.

The adequate separation between $\lambda_{\text {res_S }}$ and $\lambda_{\text {res_C }}$ must be sustained for the successful operation of the multi-purpose sensor. For that reason, we have plotted the transmission spectrum of the sensor for $L=280-300 \mathrm{~nm}$ and $R=300-350 \mathrm{~nm}$, as shown in Figure 6. The remaining geometric parameters such as $g$ and $g_{1}$ are fixed at $20 \mathrm{~nm}$ and $10 \mathrm{~nm}$, respectively. In the case when $L=R=300 \mathrm{~nm}, \lambda_{\text {res_S }}$ and $\lambda_{\text {res_C }}$ overlap each other at $1036 \mathrm{~nm}$, which is not a desirable situation for a multi-purpose sensing device. This is why an ample difference between the effective length of the cavities is crucial to attain two separate dips. For pronounced resonance dips, $L$ and $R$ are maintained at $280 \mathrm{~nm}$ and $340 \mathrm{~nm}$, respectively, which provides $\lambda_{\text {res_S }}=985 \mathrm{~nm}$ and $\lambda_{\text {res_C }}=1145 \mathrm{~nm}$, respectively. This, in turn, determines the free spectral range of $160 \mathrm{~nm}$.

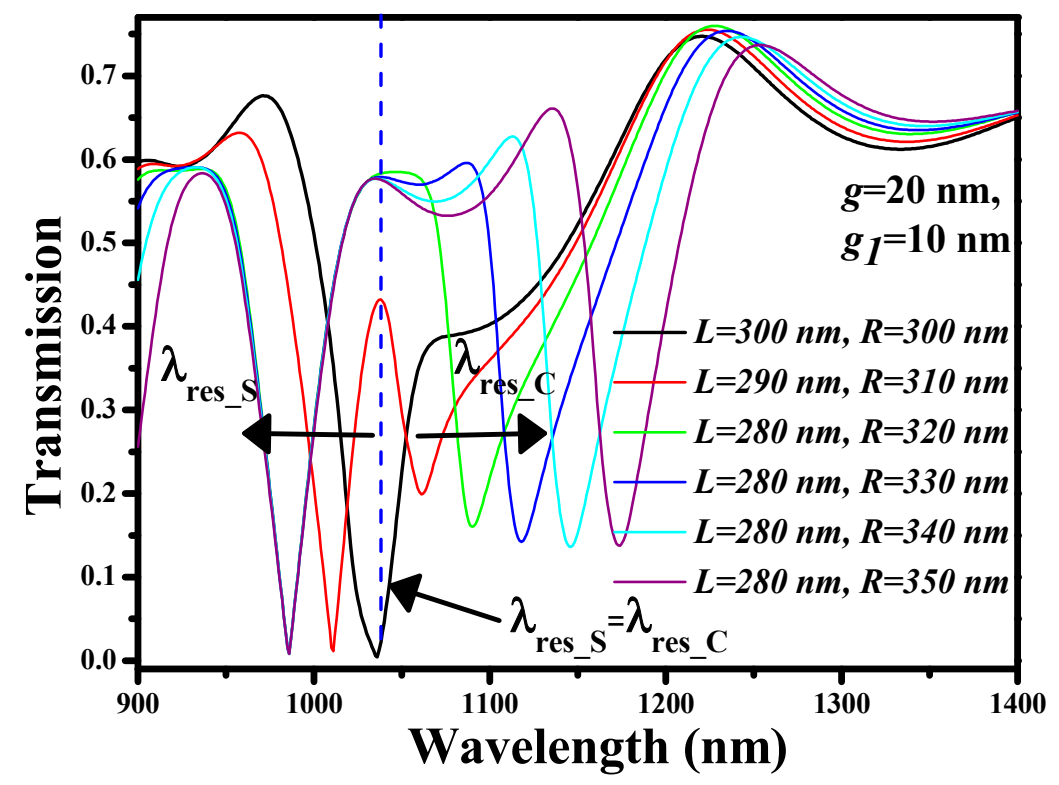

Figure 6. Transmission spectrum of square and circular cavity of various side lengths. The dotted blue line indicates the overlapping of $\lambda_{\text {res_s }}$ and $\lambda_{\text {res_C. }}$. 


\section{Modes of Operation, Results, and Discussion}

The geometric parameters of the device are optimized in Section 3 and utilized for further sensing performance. Parameters such as $L, R, g, g_{1}$, and $W$ are maintained at $280 \mathrm{~nm}, 340 \mathrm{~nm}, 20 \mathrm{~nm}, 10 \mathrm{~nm}$, and $50 \mathrm{~nm}$, respectively. Here, we have considered three distinct scenarios in which the sensor can be employed. The first considered case is a biosensor. The square cavity is filled with a dielectric material $(n=1.33)$. As a result, $\lambda_{\text {res_s }}=985 \mathrm{~nm}$ is obtained. The ambient temperature is maintained at $10^{\circ} \mathrm{C}$; accordingly, the refractive index of PDMS material is preserved at 1.4131, which give rise to $\lambda_{\text {res_C }}$ at $1145 \mathrm{~nm}$. The refractive index of the analytes is varied between 1.33 and 1.40 , which brings a redshift in $\lambda_{\text {res_s, }}$ as shown in Figure $7 a$. It is worth noting that $\lambda_{\text {res_C }}$ is unaffected throughout this sensing process.

In the second case, the proposed device is employed as a temperature sensor and the square cavity is filled with a dielectric material $(n=1.33)$. The analysis is performed for the temperature range of $10-90{ }^{\circ} \mathrm{C}$. As the ambient temperature increases, the refractive index of the PDMS material decreases, which brings a change in the effective refractive index of the SPP mode. Consequently, a blueshift is observed in $\lambda_{\text {res_C, }}$, as shown in Figure $7 \mathrm{~b}$. Once again, one can see that there is no shift in the position of $\lambda_{\text {res_s}}$. This confirms that the proposed device can be employed for multipurpose sensing applications.

In the third case, the device is employed for the simultaneous detection of analytes and temperature. The refractive index of the analytes and ambient temperature are varied at the same time from 1.33 to 1.40 and 10 to $90^{\circ} \mathrm{C}$, respectively. As a result, $\lambda_{\text {res_s }}$ and $\lambda_{\text {res_C }}$ perform a redshift and blueshift, respectively. Both the resonance dip spectrums are well separated, which does not create any ambiguity in determining the shift in the resonance wavelength, as shown in Figure 7c.

The normalized $\mathrm{H}$-field distributions corresponding to the case when resonance occurs at one of sensors and to the case where no resonance occurs are plotted in Figure 8. In the first case of the square resonator, acting as a biosensor, the SPP mode is confined in the cavity at the resonance wavelength $\lambda_{\text {res_S }}=985 \mathrm{~nm}$, as shown in Figure 8a. This resonance dip of the transmission spectrum must be traced while the biological samples will be analyzed with this sensor. In the second case, the H-field distribution at $\lambda_{\text {res_}} S=1145 \mathrm{~nm}$ is plotted, which shows the confinement of SPP mode in the circular cavity, as shown in Figure $8 \mathrm{~b}$. As the temperature of the ambient medium increases, the refractive index of the PDMS layer varies, resulting in a blueshift of the resonant wavelength $\lambda_{\text {res_C. }}$. For temperature sensing applications, a shift in $\lambda_{\text {res_C }}$ must be tracked. In the third case, the $\mathrm{H}$-field is plotted at $\lambda=1250 \mathrm{~nm}$, which corresponds with the non-resonant transmission of both resonators, as shown in Figure 8c.

To verify the sensor performance, two important factors should be considered, namely the sensitivity and the figure of merit. The sensitivity $(S)$ of the dual-purpose device can be calculated using the following expressions.

$$
S_{B}=\frac{\Delta \lambda_{\text {res_S }}}{\Delta n} ; S_{T}=\frac{\Delta \lambda_{\text {res_C }}}{\Delta T}
$$

where $S_{B}$ and $S_{T}$ are the sensitivity of biosensor and temperature sensor, respectively. $\Delta \lambda_{\text {res_S } S}, \Delta \lambda_{\text {res_C }}, \Delta n$, and $\Delta T$ are the change in the resonance wavelength of a square cavity, change in the resonance wavelength of a circular cavity, change in the refractive index, and temperature change, respectively. Figure $9 \mathrm{a}, \mathrm{b}$ shows the $\Delta \lambda_{\text {res_S } S}$ and $\Delta \lambda_{\text {res_C }}$ plot versus RIU and $\mathrm{T}\left({ }^{\circ} \mathrm{C}\right)$, respectively. In the case of biosensors, $\Delta \lambda_{\text {res_S }}$ shows an upward linear trend with an increase in RIU. The effective refractive index of the SPP mode increases with an increase in the local refractive index which leads to the shift of $\lambda_{\text {res_s }}$ to a higher wavelength. However, $\Delta \lambda_{\text {res_C }}$ shows a downward linear trend with an increase in $\mathrm{T}\left({ }^{\circ} \mathrm{C}\right)$. This is owing to the reduction in the refractive index of the PDMS material under the influence of increasing temperature. $S_{B}$ and $S_{T}$ are determined at $700 \mathrm{~nm} / \mathrm{RIU}$ and $-0.35 \mathrm{~nm} /{ }^{\circ} \mathrm{C}$, respectively, as shown in Figure $9 \mathrm{c}, \mathrm{d}$. The sensitivities acquired in the proposed sensor design are quite promising for such simple cavities and dual functionality. 

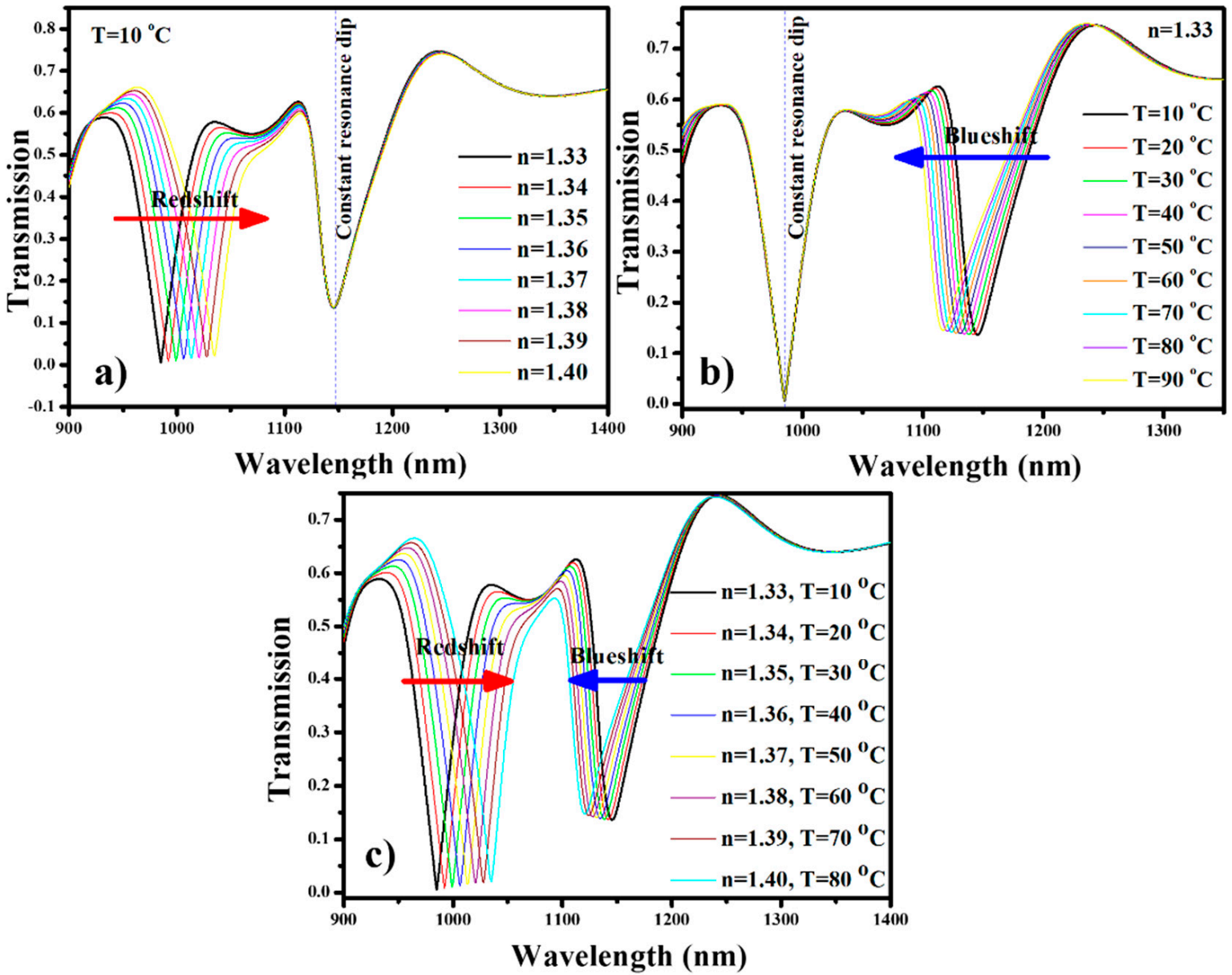

Figure 7. Transmission spectrum of the sensing device when employed as (a) biosensor, (b) temperature sensor, and (c) biosensor and temperature sensor.

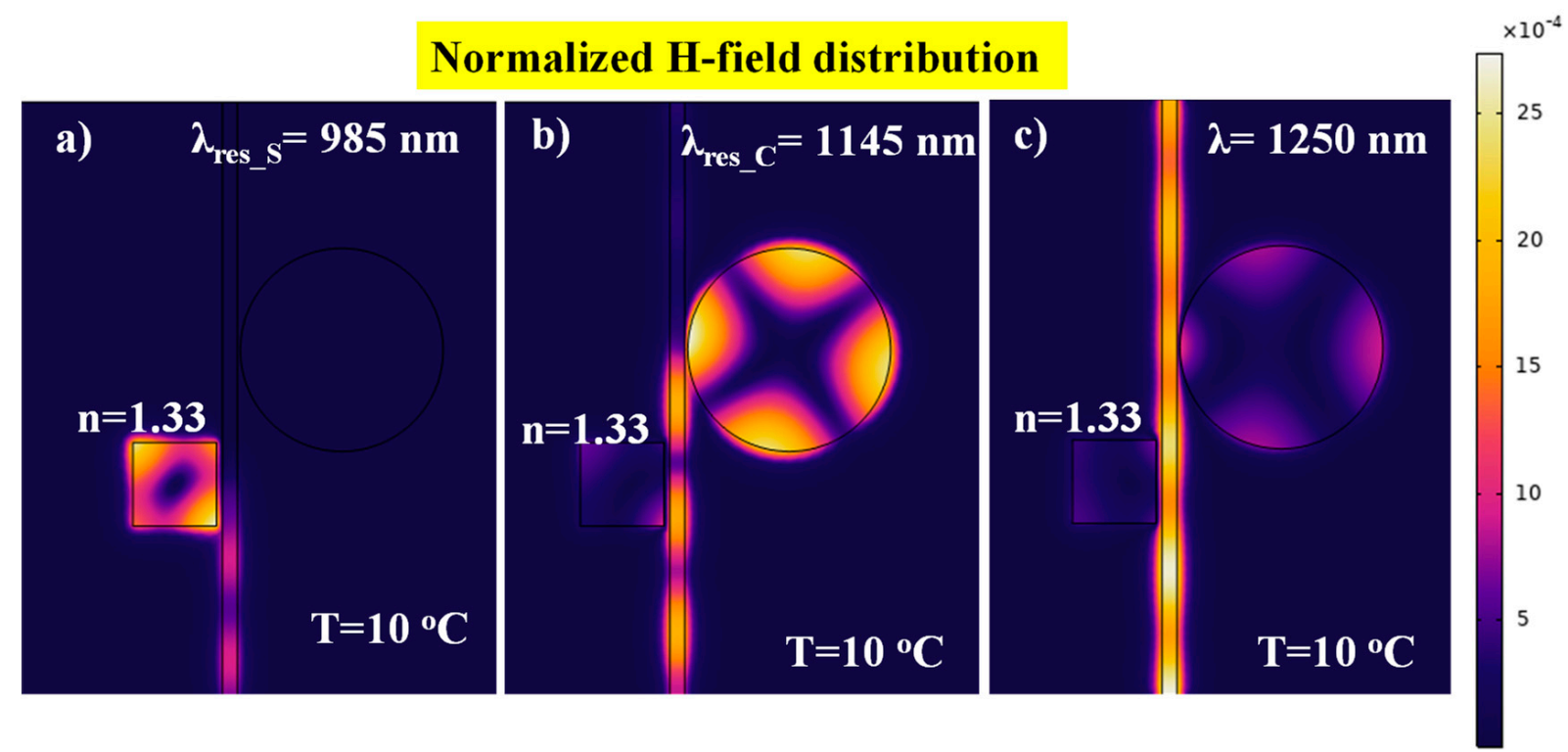

Figure 8. Normalized H-field distribution at (a) $\lambda_{\text {res_S }}=985 \mathrm{~nm}$; (b) $\lambda_{\text {res_s }}=1145 \mathrm{~nm}$; and (c) non-resonant mode, $\lambda=1250 \mathrm{~nm}$. The geometric parameters used in this analysis are $W=50 \mathrm{~nm}, L=280 \mathrm{~nm}, R=340 \mathrm{~nm}, g=20 \mathrm{~nm}$, and $g_{1}=10 \mathrm{~nm}$. 

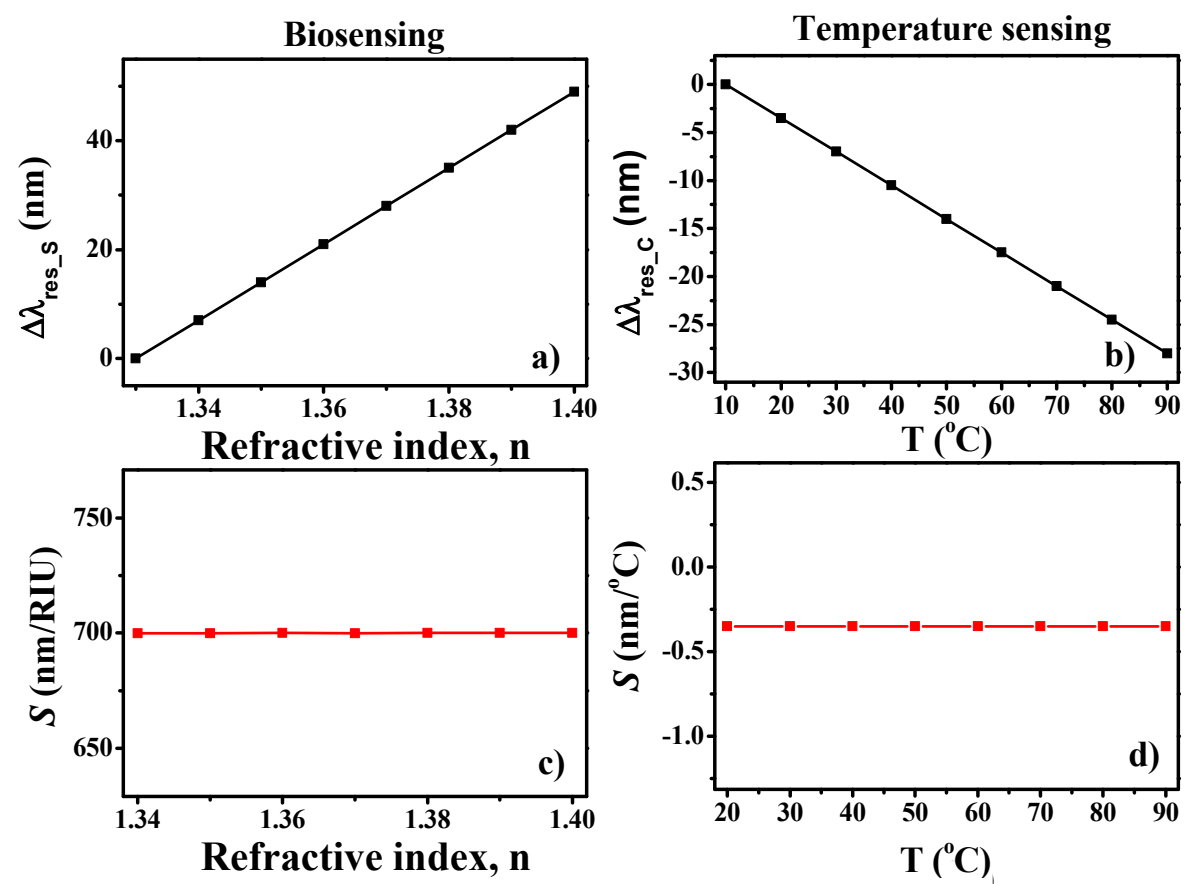

Figure 9. Spectral characteristics: (a) $\Delta \lambda_{\text {res_S }}$ versus refractive index of the analytes, (b) $\Delta \lambda_{\text {res_C }}$ versus temperature, (c) sensitivity versus refractive index of the analytes, and (d) sensitivity versus temperature.

The ability of a plasmonic sensor to quantify slight changes in the refractive index or temperature is directly proportional to $S$ and, additionally, inversely proportional to the width of the resonant feature (spectral dip) being tracked. The combination of these parameters is often referred to as the figure of merit (FOM) and can be calculated as

$$
F O M=S_{B} / F W H M \text { and } F O M=S_{T} / F W H M,
$$

where FWHM is the full width at half maximum of the respective resonance dip. The FOM for the biosensing module and temperature sensing module is around 21.9 and 0.008 , respectively.

\section{Fabrication Inaccuracies Influence Analysis}

Based on the currently available technology, it is possible to fabricate the nanostructure plasmonic sensors with the fabrication errors of \pm 5 to $\pm 10 \mathrm{~nm}$ [39]. However, inaccuracies may appear during chemical etching of the MIM waveguide patterns owing to careless handling of the etchant. In this section, two different cases of fabrication inaccuracies, i.e., under-etching and over-etching, which may be likely to appear, have been discussed. From this analysis, it will be clear that tolerant is the proposed sensor design against fabrication errors.

\subsection{Under-Etching Influence}

This situation occurs when the sample is etched for less time than it requires to fully remove the metal layer from the patterned cavity design. There can be different patterns in which inaccuracies can occur. However, here, we have considered three different points-of-inaccuracies for square cavity and three for the circular cavity. The three sides of the cavities are under-etched as a result $(\sim 30-40 \mathrm{~nm})$, and the shape is not fully squared and/or circular. This means that the effective side length is smaller than the expected one. The transmission spectrum of the under-etched sample is simulated and plotted in Figure 10a. It can be seen that $\lambda_{\text {res_s }}$ and $\lambda_{\text {res_C }}$ have shifted to a lower wavelength compared with the ones obtained in Figure 7c. There is no variation in $S_{B}$ and $S_{T}$; however, the line shape has deteriorated, which results in lower $F O M$, i.e., 
14.58 for biosensing module and 0.0088 for temperature sensing module. The $\mathrm{H}$-field distribution for under-etched square cavity and circular cavity at $\lambda_{\text {res_S }}=943 \mathrm{~nm}$ and $\lambda_{\text {res_C }}=1088 \mathrm{~nm}$ is mapped as shown in Figure $10 \mathrm{~b}, \mathrm{c}$ respectively.
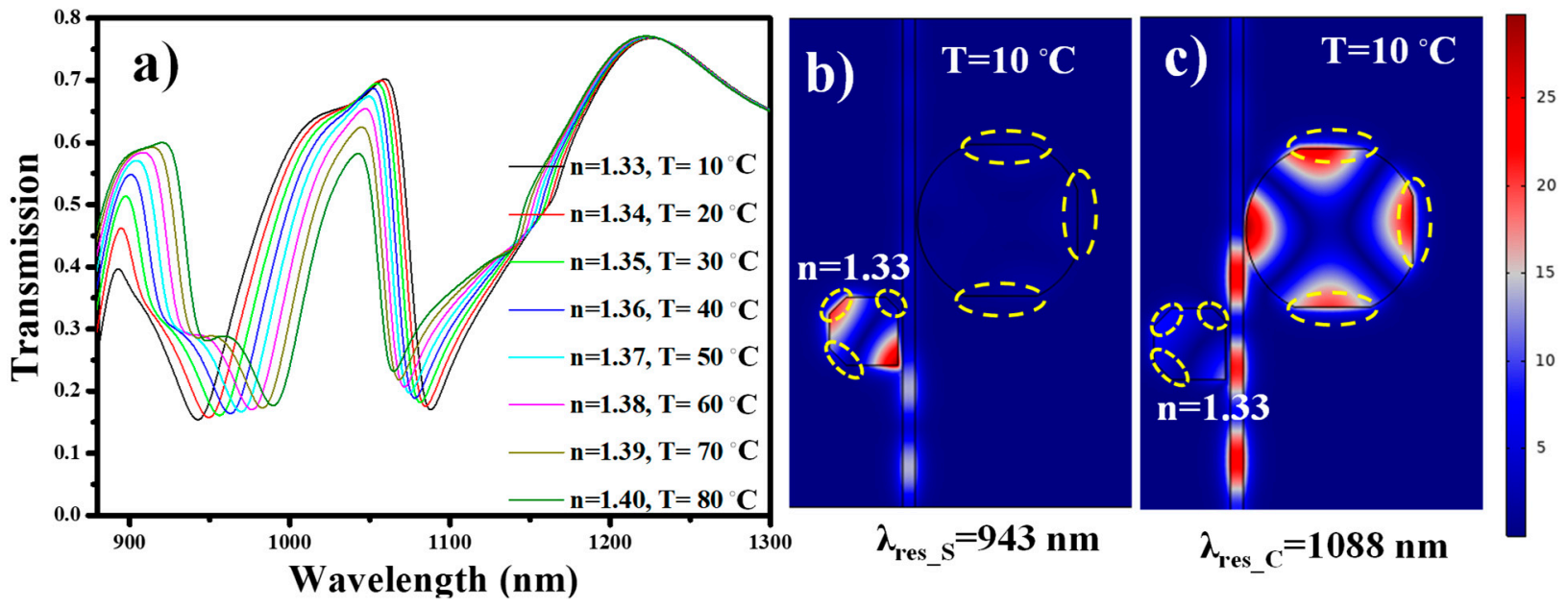

Figure 10. (a) Transmission spectrum of the over-etched device employed as biosensor and temperature sensor, (b) H-field distribution at $\lambda_{\text {res_s }}=943 \mathrm{~nm}$, and (c) H-field distribution at $\lambda_{\text {res_c }}=1088 \mathrm{~nm}$. The yellow dotted circle represents the fabrication inaccuracies.

\subsection{Over-Etching}

Here, the effect of over-etching of the cavities on the device performance was discussed. Like Section 5.1, three points-of-inaccuracies were considered at the same point; however, this time with over-etched patterns. The cavities are not a perfect shape, as was expected. This kind of situation appears when the sample is etched for a longer time than required. The transmission spectrum of the over-etched sample is simulated and plotted in Figure 11a. As the size of the cavities is larger than expected, $\lambda_{\text {res_s }}$ and $\lambda_{\text {res_c }}$ are shifted to higher wavelengths compared with those presented in Figure $7 \mathrm{c} . S_{B}$ and $S_{T}$ are not influenced and demonstrate a stable value at $700 \mathrm{~nm} / \mathrm{RIU}$ and $-0.35 \mathrm{~nm} /{ }^{\circ} \mathrm{C}$ for biosensing module and temperature sensing module, respectively. In this case, the line shape is less effected, which results in a little variation in the FOM. The resulting FOM of the biosensing and temperature sensing module is around 18.91 and 0.0045 , respectively. The H-field distribution for overetched square cavity and circular cavity at $\lambda_{\text {res_s }}=1032 \mathrm{~nm}$ and $\lambda_{\text {res_C }}=1188 \mathrm{~nm}$ is shown in Figure 11b,c, respectively.
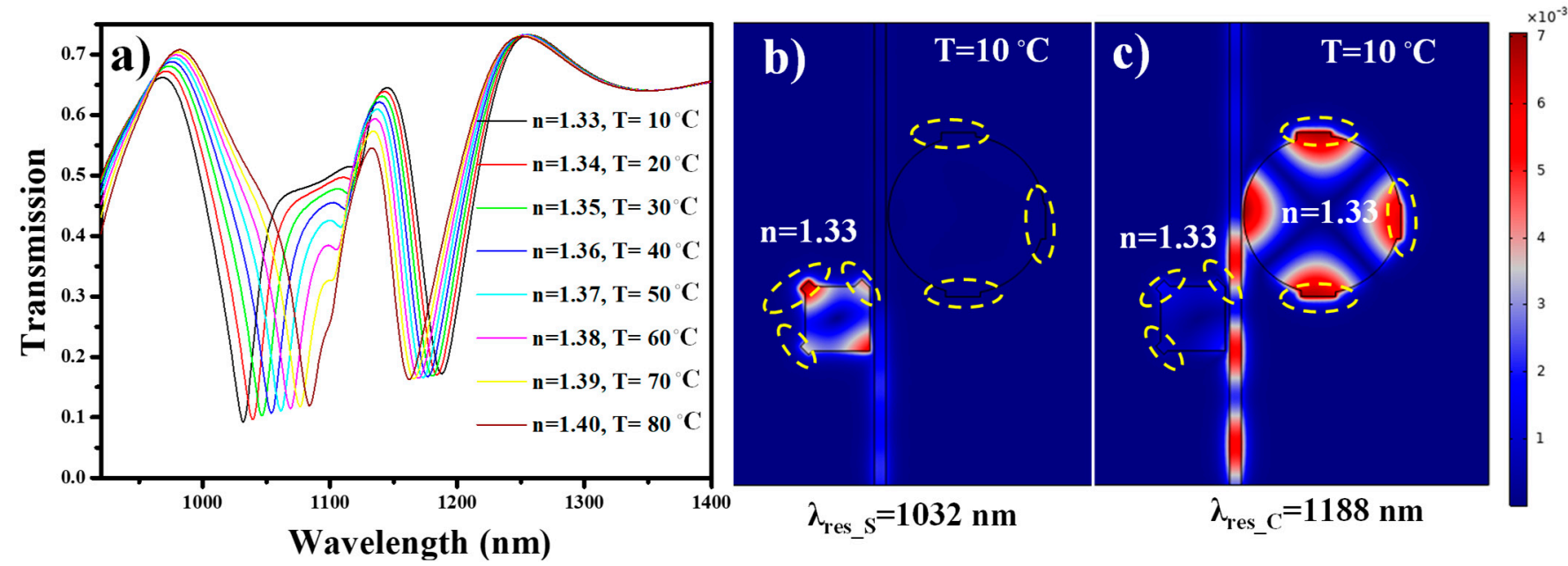

Figure 11. (a) Transmission spectrum of the under-etched device employed as biosensor and temperature sensor, (b) H-field distribution at $\lambda_{\text {res_S }}=1032 \mathrm{~nm}$, and (c) H-field distribution at $\lambda_{\text {res_C }}=1188 \mathrm{~nm}$. The yellow dotted circle represents the fabrication inaccuracies. 


\section{Limiting Factors}

Although the proposed sensor design is unique and multifunctional, it has some preventive factors that should be well-thought-out. When the sensor is employed for a single application (biosensing or temperature sensing), there will be no trouble. However, the dilemma may occur when the device is employed for the simultaneous detection of analytes and temperature. The sensor must not be exposed to extremely high or low temperatures while biosensing [44]. A high temperature can melt or damage the analytes, while a low temperature can freeze them, which can potentially cause inaccuracy in the measurement. For instance, heating a DNA solution above room temperature results in the separation of strands [45]. The temperature when half of the DNA molecule is denatured is termed as the melting temperature, which is around $50-100{ }^{\circ} \mathrm{C}$. Moreover, the melting temperature also depends on several other factors like the length of the DNA and nucleotide sequence composition.

For optimum performance, a temperature-resistant instrumental design is required, which includes temperature control of the light source, temperature control of the analytes, and the use of low-thermal-expansion-coefficient materials for all-optical components. This will be combined with an optical alignment/mechanical arrangement of everything, including the light source, lenses, prism, optical chip, chip holder, and camera, all of which will be insensitive to thermo-mechanical displacements. Moreover, reference sensor cavities can also be employed, which should be maintained at a fixed temperature and refractive index assisting in the calibration of the sensing device.

\section{Conclusions}

Herein, a plasmonic sensor based on an MIM waveguide is proposed for the simultaneous detection of biological analytes and ambient temperature. The sensor is comprised of a square and circular cavity of different effective lengths side coupled to a bus waveguide. As a result, two distinct resonance dips are obtained in the transmission spectrum. The square cavity is filled with a dielectric material such as water $(n=1.33)$ and different analytes are introduced into the cavity, which brings a redshift in the resonance wavelength, whereas the circular cavity is deposited with a thermally sensitive polymer material such as PDMS. Thanks to the high thermo-optic coefficient of the PDMS, the resonance dip related to the circular cavity performs a significant blueshift. As a result, two different functionalities are performed on the same chip independently. The bio-sensitivity and temperature sensitivity are obtained at $700 \mathrm{~nm} / \mathrm{RIU}$ and $-0.35 \mathrm{~nm} /{ }^{\circ} \mathrm{C}$, respectively. The FOM for the biosensing module and temperature sensing module is around 21.9 and 0.008 , respectively. The proposed sensor design is useful for lab-on-chip diagnostics of the bio analytes in a controlled temperature environment.

Author Contributions: Conceptualization, N.L.K., M.A.B. and S.N.K.; Methodology, N.L.K., M.A.B., S.N.K., A.K. and R.P.; Software, N.L.K., M.A.B., S.N.K., A.K. and R.P.; Validation, N.L.K., M.A.B., S.N.K., A.K. and R.P.; Formal analysis, M.A.B.; Investigation, N.L.K., M.A.B. and S.N.K.; Resources, N.L.K., M.A.B., S.N.K., A.K. and R.P.; Data curation, N.L.K., M.A.B., S.N.K., A.K. and R.P.; Writing-original draft preparation, M.A.B.; Writing—review and editing, N.L.K., M.A.B., S.N.K., A.K. and R.P.; Visualization, N.L.K., M.A.B., S.N.K., A.K. and R.P.; Supervision, N.L.K., M.A.B. and S.N.K.; Project administration, N.L.K., M.A.B., S.N.K., A.K. and R.P.; Funding acquisition, N.L.K., M.A.B., S.N.K., A.K. and R.P. All authors have read and agreed to the published version of the manuscript.

Funding: This work was financially supported by the Ministry of Science and Higher Education of the Russian Federation (the scientific state assignment No. 0777-2020-0017 of the Samara National Research University) for numerical calculations, by the FSRC "Crystallography and Photonics" of the Russian Academy of Sciences (the state task No. 007-GZ/Ch3363/26) for theoretical research, and by the Russian Science Foundation (grant No. 20-69-47110) for comparative analysis. Moreover, part of this research was done within the framework of the "Hybrid sensor platforms of integrated photonic systems based on ceramic and polymer materials" project carried out within the TEAM-NET 
programme of the Foundation for Polish Science financed by the European Union under the European Regional Development Fund, POIR.04.04.00-00-14D6/18-01.

Acknowledgments: We acknowledge the equal contribution of all the authors in the accomplishment of this work.

Conflicts of Interest: The authors declare no conflict of interest.

\section{References}

1. Butt, M.A.; Khonina, S.N.; Kazanskiy, N.L. Plasmonics: A necessity in the field of sensing-A review (invited). Fiber Integr. Opt. 2021, 40, 14-47. [CrossRef]

2. Sakai, H.; Okahisa, S.; Nakayama, Y.; Nakayama, K.; Fukuhara, M.; Kimura, Y.; Ishii, Y.; Fukuda, M. Plasmonic and electronic device-based integrated circuits and their characteristics. Solid State Electron. 2016, 125, 240-246. [CrossRef]

3. Zhang, J.; Zhang, L.; Xu, W. Surface plasmon polaritons: Physics and applications. J. Phys. D Appl. Phys. 2012, 45, 113001. [CrossRef]

4. Butt, M.A. Numerical investigation of a small footprint plasmonic Bragg grating structure with a high extinction ratio. Photonics Lett. Pol. 2020, 12, 82-84. [CrossRef]

5. Butt, M.A.; Khonina, S.N.; Kazanskiy, N.L. Ultra-short lossless plasmonic power splitter design based on metal-insulator-metal waveguide. Laser Phys. 2019, 30, 016201. [CrossRef]

6. Chao, C.-T.; Chau, Y.-F.C.; Huang, H.; Kumara, N.; Kooh, M.; Lim, C.; Chiang, H.-P. Highly sensitive and tunable plasmonic sensor based on a nanoring resonator with silver nanorods. Nanomaterials 2020, 10, 1399. [CrossRef] [PubMed]

7. Masson, J.-F. Portable and field-deployed surface plasmon resonance and plasmonic sensors. Analyst 2020, 145, 3776-3800. [CrossRef]

8. Butt, M.A.; Kazanskiy, N.L. Nanoblocks embedded in L-shaped nanocavity of a plasmonic sensor for best sensor performance. Opt. Appl. 2021, 51, 109-120.

9. Zhu, J.; Lou, J. Ultrasensitive and multifunction plasmonic temperature sensor with ethanol-sealed asymmetric ellipse resonators. Molecules 2018, 23, 2700. [CrossRef]

10. Zhu, J.; Lou, J. High-sensitivity Fano resonance temperature sensor in MIM waveguides coupled with a polymethylsiloxane-sealed semi-square ring resonator. Results Phys. 2020, 18, 103183. [CrossRef]

11. Tian, M.; Lu, P.; Chen, L.; Liu, D.; Lv, C. All-optical switching in MIM waveguide resonator with an outer portion smooth bend structure containing nonlinear optical materials. Opt. Commun. 2012, 285, 4562-4566. [CrossRef]

12. Shibayama, J.; Kawai, H.; Yamauchi, J.; Nakano, H. Analysis of a 3D MIM waveguide-based plasmonic demultiplexer using the TRC-FDTD method. Opt. Commun. 2019, 452, 360-365. [CrossRef]

13. Chau, Y.-F.C.; Chao, C.-T.C.; Chaing, H.-P. Ultra-broad bandgap metal-insulator-metal waveguide filter with symmetric stubs and defects. Results Phys. 2020, 17, 103116. [CrossRef]

14. Butt, M.A.; Kazanskiy, N.L.; Khonina, S.N. Highly sensitive refractive index sensor based on plasmonic Bow Tie configuration. Photonic Sens. 2020, 10, 223-232. [CrossRef]

15. Zhu, J.; Wu, C. Optical refractive index sensor with Fano resonance based on original MIM waveguide structure. Results Phys. 2021, 21, 103858.

16. Wang, Y.; Gao, B.; Zhang, K.; Yuan, K.; Wan, Y.; Xie, Z.; Xu, X.; Zhang, H.; Song, Q.; Yao, L.; et al. Refractive index sensor based on leaky resonant scattering of single semiconductor nanowire. ACS Photon. 2017, 4, 688-694. [CrossRef]

17. Teng, C.; Yu, F.; Ding, Y.; Zheng, J. Refractive index sensor based on multi-mode plastic optical fiber with long period grating. Proc. SPIE 2017, 10231, 102311M.

18. Xu, D.-X; Vachon, M.; Densmore, A.; Ma, R.; Janz, S.; Delâge, A.; Lapointe, J.; Cheben, P.; Schmid, J.H.; Post, E.; et al. Real-time cancellation of temperature induced resonance shifts in SOI wire waveguide ring resonator label-free biosensor arrays. Opt. Express 2010, 18, 22867-22879. [CrossRef]

19. Talebifard, S.; Schmidt, S.; Shi, W.; Wu, W.; Jaeger, N.A.F.; Kwok, E.; Ratner, D.M.; Chrostowski, L. Optimized sensitivity of silicon-on-insulator (SOI) strip waveguide resonator sensor. Biomed. Opt. Express 2017, 8, 500-511. [CrossRef]

20. Khonina, S.N.; Kazanskiy, N.L.; Butt, M.A.; Kazmierczak, A.; Piramidowicz, R. Plasmonic sensor based on metal-insulator-metal waveguide square ring cavity filled with functional material for the detection of CO2 gas. Opt. Express 2021, 29, 16584. [CrossRef]

21. Liu, Y.; Ma, Y. One-dimensional plasmonic sensors. Front. Phys. 2020, 8, 312. [CrossRef]

22. Butt, M.A.; Kazanskiy, N.L.; Khonina, S.N. Highly integrated plasmonic sensor design for the simultaneous detection of multiple analytes. Curr. Appl. Phys. 2020, 20, 1274-1280. [CrossRef]

23. Kong, Y.; Wei, Q.; Liu, C.; Wang, S. Nanoscale temperature sensor based on Fano resonance in metal-insulator-metal waveguide. Opt. Commun. 2017, 384, 85-88. [CrossRef]

24. Rahmatiyar, M.; Afsahi, M. Design of a refractive index plasmonic sensor based on a ring resonator coupled to a MIM waveguide containing tapered defects. Plasmonics 2020, 15, 2169-2176. [CrossRef]

25. Yang, X.; Hua, E.; Wang, M.; Wang, Y.; Wen, F.; Yan, S. Fano resonance in a MIM waveguide with two triangle stubs coupled with a split-ring nanocavity for sensing application. Sensors 2019, 19, 4972. [CrossRef] [PubMed] 
26. Zhang, Y.; Cui, M. Refractive index sensor based on the symmetric MIM waveguide structure. J. Electron. Mater. 2019, 48, 1005-1010. [CrossRef]

27. Zhao, X.; Zhang, Z.; Yan, S. Tunable Fano resonance in asymmetric MIM waveguide structure. Sensors 2017, 17, 1494. [CrossRef] [PubMed]

28. Xie, Y.; Huang, Y.; Xu, W.; Zhao, W.; He, C. A plasmonic temperature-sensing structure based on dual laterally side-coupled hexagonal cavities. Sensors 2016, 16, 706. [CrossRef]

29. Chen, F.; Zhang, H.; Sun, L.; Li, J.; Yu, C. Temperature tunable Fano resonance based on ring resonator side coupled with a MIM waveguide. Opt. Laser Technol. 2019, 116, 293-299. [CrossRef]

30. Fan, J.; Zhang, J.; Lu, P.; Tian, M.; Xu, J.; Liu, D. A single-mode fiber sensor based on core-offset inter-modal interferometer. Opt. Commun. 2014, 320, 33-37. [CrossRef]

31. Srivastava, T.; Das, R.; Jha, R. Highly sensitive plasmonic temperature sensor based on Photonic Crystal surface plasmon waveguide. Plasmonics 2013, 8, 515-521. [CrossRef]

32. Lotters, J.C.; Olthuis, W.; Veltink, P.H.; Bergveld, P. The mechanical properties of the rubber elastic polymer polydimethylsiloxane for sensor applications. J. Micromechanics Microengineering 1997, 7, 145. [CrossRef]

33. Shi, H.; Yan, S.; Yang, X.; Wu, X.; Wu, W.; Hua, E. A nanosensor based on a metal-insulator-metal bus waveguide with a stub coupled with a racetrack ring resonator. Micromachines 2021, 12, 495. [CrossRef] [PubMed]

34. Hu, F.; Chen, F.; Zhang, H.; Sun, L.; Yu, C. Sensor based on multiple Fano resonances in MIM waveguide resonator system with silver nanorod-defect. Optik 2021, 229, 166237. [CrossRef]

35. Bahri, H.; Mouetsi, S.; Hocini, A.; Salah, H.B. A highly sensitive sensor using MIM waveguide coupled with a rectangular cavity with Fano resonance. Opt. Quantum Electron. 2021, 53, 332. [CrossRef]

36. Rakhshani, M.R. Refractive index sensor based on concentric triple racetrack resonators side-coupled to metal-insulator-metal waveguide for glucose sensing. J. Opt. Soc. Am. B 2019, 36, 2834-2842. [CrossRef]

37. Huang, B.; Meng, H.; Wang, Q.; Wang, H.; Zhang, X.; Yu, W.; Tan, C.; Huang, X.; Wang, F. Plasmonic-induced transparency and slow-light effect based on stub waveguide with nanodisk resonator. Plasmonics 2016, 11, 543-550. [CrossRef]

38. Kazanskiy, N.L.; Butt, M.A.; Khonina, S.N. Nanodots decorated MIM semi-ring resonator cavity for biochemical sensing. Photonics Nanostructures Fundam. Appl. 2020, 42, 100836. [CrossRef]

39. Manfrinato, V.R.; Zhang, L.; Dong, S.; Duan, H.; Hobbs, R.G.; Stach, E.A.; Berggren, K.K. Resolution limits of electron-beam lithography toward the atomic scale. Nano Lett. 2013, 13, 1555-1558. [CrossRef]

40. Sia, P.D. Overview of Drude-Lorentz type models and their applications. Nanoscale Syst. Math. Model. Theory Appl. 2014, 3, 1-13.

41. Butt, M.A.; Khonina, S.N.; Kazanskiy, N.L. Metal-insulator-metal nano square ring resonator for gas sensing applications. Waves Random Complex Media 2021, 31, 146-156. [CrossRef]

42. Kazanskiy, N.L.; Khonina, S.N.; Butt, M.A. Plasmonic sensors based on Metal-insulator-metal waveguides for refractive index sensing applications: A brief review. Phys. E 2020, 117, 113798. [CrossRef]

43. Zhu, Z.; Liu, L.; Liu, Z.; Zhang, Y.; Zhang, Y. Surface-plasmon-resonance-based optical-fiber temperature sensor with high sensitivity and high figure of merit. Opt. Lett. 2017, 42, 2948-2951. [CrossRef] [PubMed]

44. Haslacher, H.; Szekeres, T.; Gerner, M.; Ponweiser, E.; Repl, M.; Wagner, O.F.; Perkmann, T. The effect of storage temperature fluctuations on the stability of biochemical analytes in blood serum. Clin. Chem. Lab. Med. 2017, 55, 974-983. [CrossRef] [PubMed]

45. Wartell, R.M.; Benight, A.S. Thermal denaturation of DNA molecules: A comparison of theory with experiment. Phys. Rep. 1985, 126, 67-107. [CrossRef] 